\title{
Modelling and simulation of online partial discharge measurement for medium voltage power cable
}

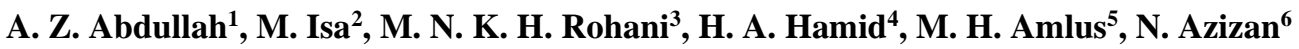 \\ 1,2,3,4 High Voltage Transient \& Insulation Health (HVTrans) Group, Centre of Excellence for Renewable Energy \\ (CERE), School of Electrical System Engineering, Universiti Malaysia Perlis (UniMAP), 02600 Arau, Perlis, Malaysia \\ ${ }^{5,6}$ Faculty Engineering Technology, Universiti Malaysia Perlis (UniMAP) Padang Besar Perlis, Malaysia
}

\begin{tabular}{l} 
Article Info \\
\hline Article history: \\
Received Nov 15, 2019 \\
Revised Jan 4, 2019 \\
Accepted Feb 6, 2020 \\
\hline
\end{tabular}

\section{Keywords:}

Measurement

Modelling

Partial discharge

Power cable

Rogowski coil

Simulink

\begin{abstract}
This paper presents the modelling of the online partial discharge (PD) measurement of the medium voltage (MV) power cable. Recently, PD monitoring trends are rapidly increasing due to high demand on reliable systems. Degradation are mainly due to the presence of PD in the high voltage power equipment used. PD measurement is therefore a highly recommended task to early detection of the degradation insulation for high voltage (HV) equipment in order to avoid breakdowns. Real network modelling is necessary to improvise system design in order to find the efficiency in a real power system network. In this paper, modelling focuses on a real distribution network by applying Rogowski coil (RC) as a detection sensor to trigger PD activity. The simulation is performed to determine the functionality and reliability of the system with the RC application in the network. The analysis is performed in the ATP-EMTP and MATLAB Simulink software environments. In addition, this paper contributed to justify the approach of a simplified PD sensor and measurement system. This PD measurement system provides a complete solution in the context of condition-oriented monitoring for the ability to apply the RC to trigger PD activity in the power distribution network.
\end{abstract}

This is an open access article under the CC BY-SA license.

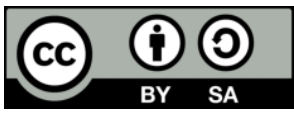

Corresponding Author:

A. Z. Abdullah,

School of Electrical System Engineering,

Universiti Malaysia Perlis (UniMAP),

Kampus Pauh Putra, 02600 Arau, Perlis, Malaysia.

Email: zaidiabdullah@unimap.edu.my

\section{INTRODUCTION}

The condition monitoring system plays an important role in ensuring that the electrical supply network operates efficiently. The electrical supply worldwide runs simultaneously at 24 hours and 7 days a week, particularly on the side of MV. As a result, various components of MV electrical power face environmental stress [1]. Condition-based monitoring of the power system needs the best part and equipment infrastructure that is reliable when efficient maintenance is performed [2]. Early information on possible breakdowns in online mode is considered to be crucial, particularly for PD in HV equipment [3-4]. As a result, utility providers continue to strive to find ways to increase the efficiency of the electricity supply's continuity [5-7].

One of the key components is the MV underground power cable for distribution networks. This element is usually subjected to stress, including electrical, thermal, mechanical and environmental stress, which contributes significantly to the deterioration of its insulation [8-9]. Degradation insulation in MV underground cable is highly proven by PD activities [3]. PD is a localized discharge that only partially 
bridges the insulation between the conductor and may or may not occur adjacent to the conductor [10]. The high precision of the PD measurement system is essential for capturing PD activity as it occurs in the short duration of current pulses, usually for only a few nanoseconds [11-13, 14]. PD usually occurs before the breakdown indicates that PD monitoring can be a good system to install, with reference to data from [15]. The data collected through the PD monitoring system can also keep the insulation system stable in real-time. It is therefore possible to improve reliability, performance, equipment security and costs. The traditional method is to use off-line measurement where the routine is disrupted. It will be more economical to inspect the equipment without interrupting the whole system. Online PD assessment is the best way to ensure the optimum quality of PD analysis [2,7,16,17]. The standard PD measurement refers to IEC 60270 [18], which provided a complete measure requirement. The main advantage of the PD measurement is the early detection of PD activity, which can prevent the equipment from breaking down [19-21].

At present, the traditional PD sensors are saturated, tremendous, bulky, low sensitivity, and high cost compared to Rogowski coils (RC), such as High-Frequency Current Transformer (HFCT) and Coupling Capacitor (CC)[22]. The PD behavior will be measured by the current pulse when the RC is clamped around the supply cable $[6,23]$. Whereas online measurements also have drawbacks, such as the need to collect data manually and take an enormous amount of time and human power [24-25]. Over the last few years, PD measurement has attracted increasing attention due to the high efficiency and reliability of the system[26-27]. In this paper, therefore, the modelling and simulation of online PD measurements for medium voltage power cables in order to identify the capability of the RC sensor performed in a real network has been successful with the significant PD waveform. The apparent conversion of the charge was also introduced, indicating a good result of the simulation. It is therefore clear that the RC sensor developed is suitable for use on-site with high advantages for the user.

\section{RESEARCH METHOD}

Although realistic findings from PD measurements and test samples are the main source for research and analysis of the PD phenomenon, modelling and simulation often play an important role in PD studies. A reliable PD model can save time for realistic experiments that can be performed over hours or days and can predict insulation life without repetitive, long term tests. This modelling system is developed by reference to the standard IEC 60270. The RC [28] is used as a modelling measurement sensor. Figure 1(a) shows the complete model of a $70 \mathrm{~km}$ power distribution network using the J-Marti model, and Figure 1(b) shows the three phase RC model using the EMTP-ATP software. The network consists of a $33 \mathrm{kV}$ power source, a 33/11 kV step-down transformer, a $300 \mathrm{~mm} 2$ XLPE underground cables and loads. The network is designed on the basis of a real network to justify the ability of the RC sensor to detect PD activity.

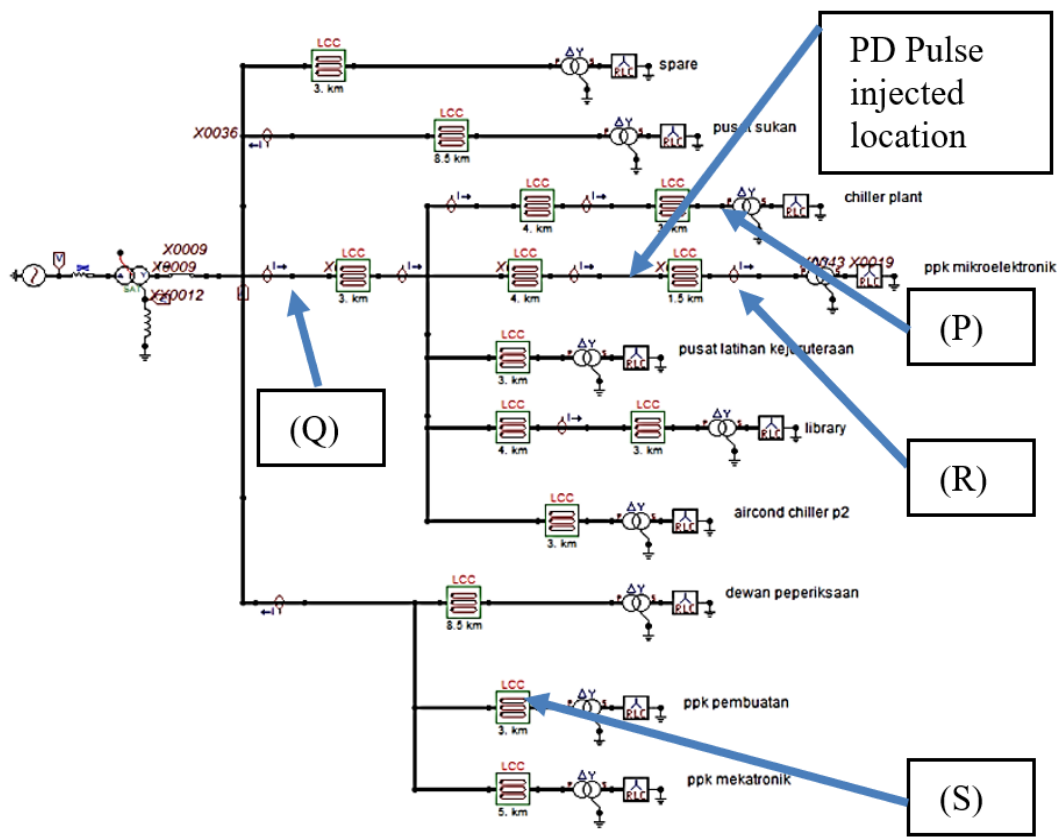

(a)

Figure 1. (a) Model of $70 \mathrm{~km}$ of real power distribution network using EMTP-ATP 


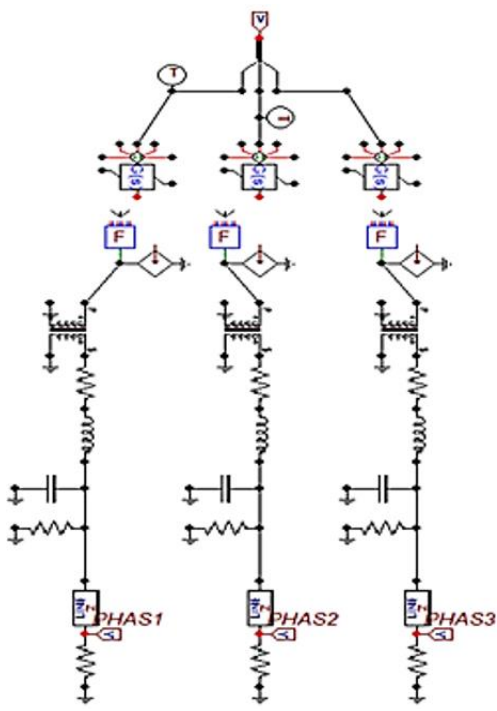

(b)

Figure 1. (b) Model of Rogowski coil for a three-phase system (continue)

The RC sensor model, as shown in Figure 1(b), has a very large frequency response and there is no conductive coupling between the coil sensors and the test element. Furthermore, the installation of the coil does not require the grounding leads of the test items to be disconnected and thus becomes a non-intrusive sensor which is very useful for on-site measurement online. The PD pulse model, as shown in Figure 2, was designed on the basis of the specific parameters indicated in Table 1 and used in the Fortran expressions by the implemented equation (1). The simulation is then communicated to the feeder as a TACS-controlled current source type 60 to generate multiple PD pulse [28]. The PD pulse is injected into the network at leg four as shown in Figure 1(a), while the RC model is connected to the points $\mathrm{P}(11 \mathrm{~km}), \mathrm{Q}(7 \mathrm{~km}), \mathrm{R}(1.5 \mathrm{~km})$ and $S(15.5 \mathrm{~km})$ from the PD pulse to find the output trigger.

$$
i(t)=A\left[10^{-1.3 \frac{\left(t-t_{0}\right)}{\tau}}-10^{-\frac{2.2\left(t-t_{0}\right)}{\tau}}\right]
$$

Where: $\mathrm{A}$ is the amplitude of the PD pulse $t_{o}$ is the time of PD occurrence $\tau$ is the damping factor of the PD pulse

Table 1. Parameter PD pulse

\begin{tabular}{cccc}
\hline No & $\mathrm{A}(\mathrm{mA})$ & $\mathrm{t}_{\mathrm{o}}(\mu \mathrm{s})$ & $\tau(\mu \mathrm{s})$ \\
\hline 1 & 14.83 & 30 & 0.08 \\
2 & 13.50 & 60 & 0.08 \\
3 & 12.75 & 90 & 0.08 \\
\hline
\end{tabular}

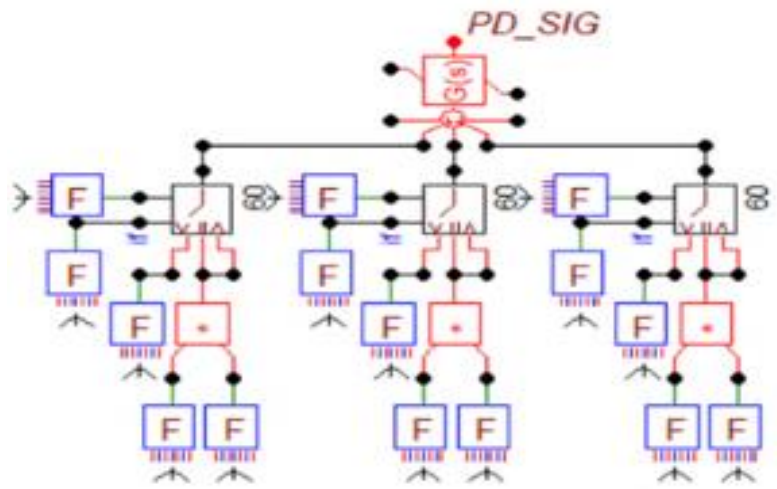

Figure 2. The model of PD pulse 


\subsection{Apparent charge conversion}

The captured PD data is primarily an oscillatory voltage pulse that needs to be processed in order to obtain PD characteristics such as peak value, apparent charge, phase position, repeat rate, and PD energy. The apparent charge $q(t)$ enters into the system due to PDs is given as:

$$
q(t)=\int i(t) d t=-\frac{1}{M} \iint \operatorname{Vout}(t) d t^{2}
$$

Where: $\operatorname{Vout}(t)$ is the oscillating voltage appearing at the output terminals of the sensor

$M$ is the mutual inductance of the RC, which is taken as $200 \mathrm{nH}$ in this work

$i(t)$ is the current flowing in the conductor due to PDs

IEC 60270 and IEEE standard states that the standard unit for measuring PD signal uses an apparent charge instead of voltage[29]. Regular modeling and measurement only produce output in $\mathrm{mV}$ unit. It is therefore necessary to convert to a charging unit. Using the above steps and the mathematical expression of the equation (2), the following Simulink model was modelled, as shown in Figure 3, to measure the PD magnitude of the load unit by the RC. The input (data3) is taken from simulation results in previous work, as mention in modelling of the measurement, and from the integral process performed to obtain the charging part. The filtering process uses a Butterworth type referring to the suggestion of previous researchers [30]. The mutual inductance of the coil is independent of its position around the cable [30]. The constant value of $M(200 \mathrm{nH})$ is therefore used for calculations and modelling.

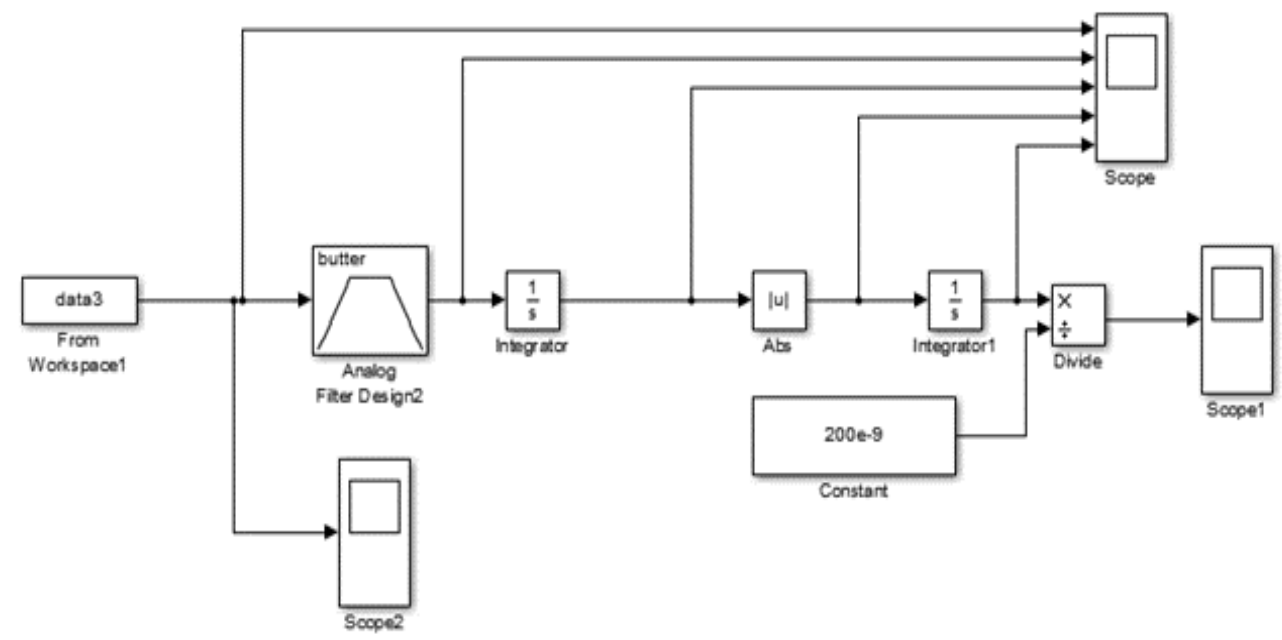

Figure 3. modelling for PD measurement in charge unit using MATLAB simulink

\section{SIMULATION RESULTS}

Simulation is performed in EMTP-ATP and MATLAB Simulink for both measurement and conversion. The model was run several times at each location of the $\mathrm{RC}$ used, and the highest and stable values were taken into account. It was set to inject the PD pulse with $3.2 \mathrm{~V}$, as shown in Figure 4 into the network location, and was repeated at least 3 times at each detected location to ensure that a correct PD pattern was captured.

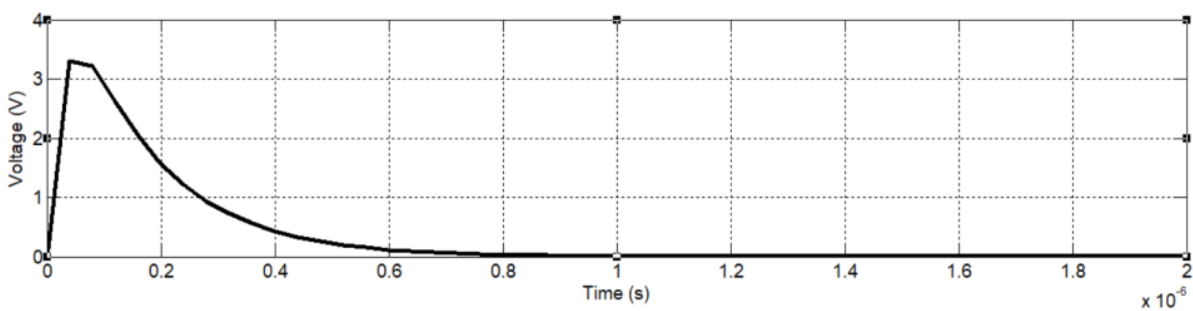

Figure 4. Output for the PD pulse model 
As a result, the PD signal is present at all measured locations $\mathrm{P}, \mathrm{Q}, \mathrm{R}$ and $\mathrm{S}$ with significant value, which is capable of clarifying the quality and distance of the PD detected from the PD source. The result shows the amplitude of the PD signal detected in the time domain while the result of the Fast Fourier Transform (FFT) is the frequency response on the PD signal. The simulation results of PD measurements are shown in Figures 5-8 and summarised in Table 2. It is shown that the PD signal is detected at the highest amplitude of $13.13 \mathrm{mV}$ and 0.0127 in FFT at location R, as shown in Figure 5. This means that the PD signal detected is closest to the PD source. While the amplitude value at location $\mathrm{S}$ is the lowest in Figure 8 with only $0.5442 \mathrm{mV}$, since the RC distance from the PD source is very far $(15.5 \mathrm{~km})$ compared to location $\mathrm{P}$ $(7 \mathrm{~km})$ with $1.089 \mathrm{mV}$ and location Q $(11 \mathrm{~km})$ with $1.089 \mathrm{mV}$. The FFT result represents the frequency response of the RC to the PD signal. Most of FFT result shows in range more than $10 \mathrm{MHz}$ fulfil the standard PD characteristic. This result can lead to the conclusion that RC sensors can be used in real networks capable of showing very significant trigger value, and that data can also be used to determine the exact location of the PD.
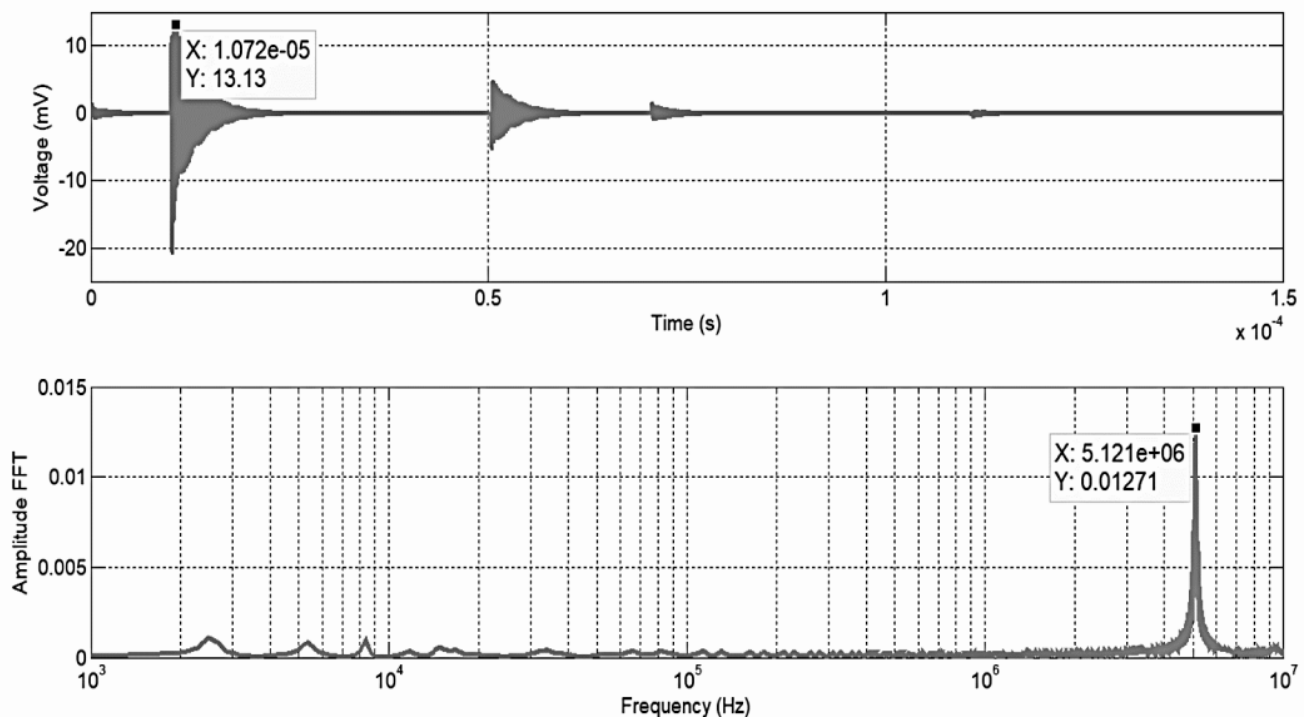

Figure 5. PD signal in time and frequency domain detected at point $\mathrm{R}(1.5 \mathrm{~km})$
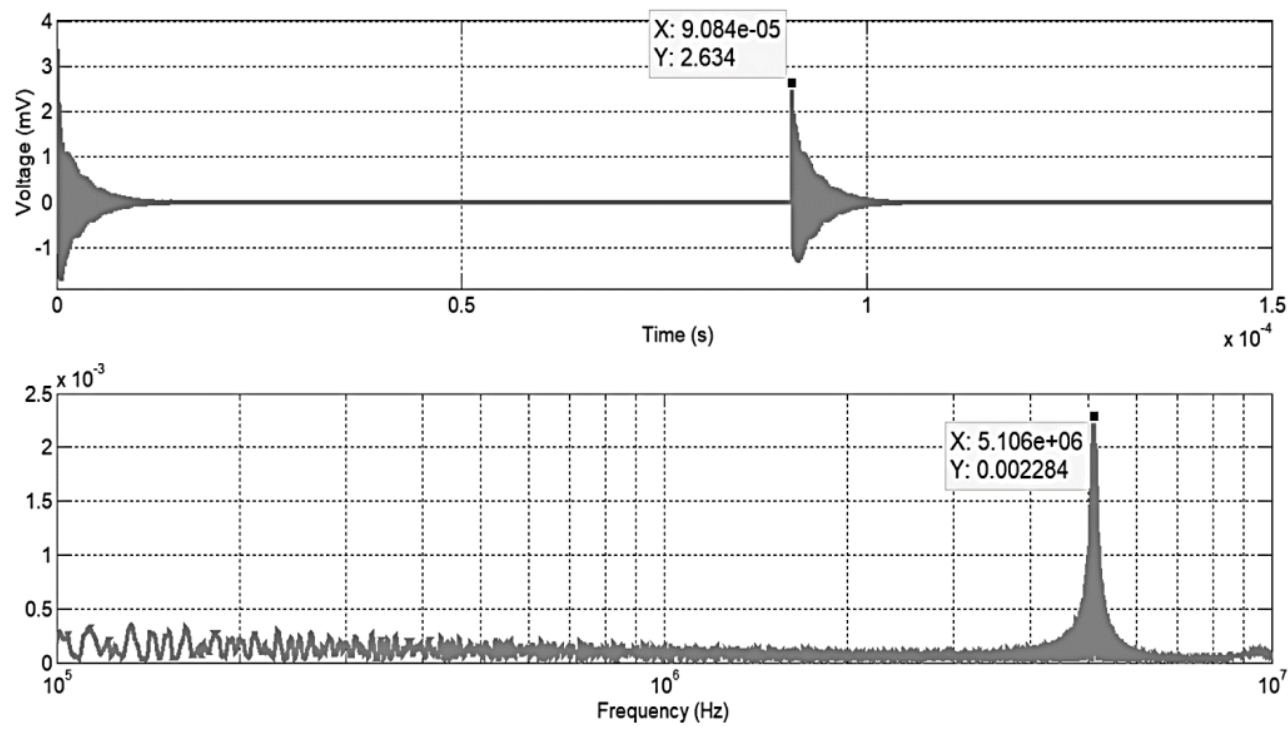

Figure 6. PD signal in time and frequency domain detected at point Q $(11 \mathrm{~km})$ 

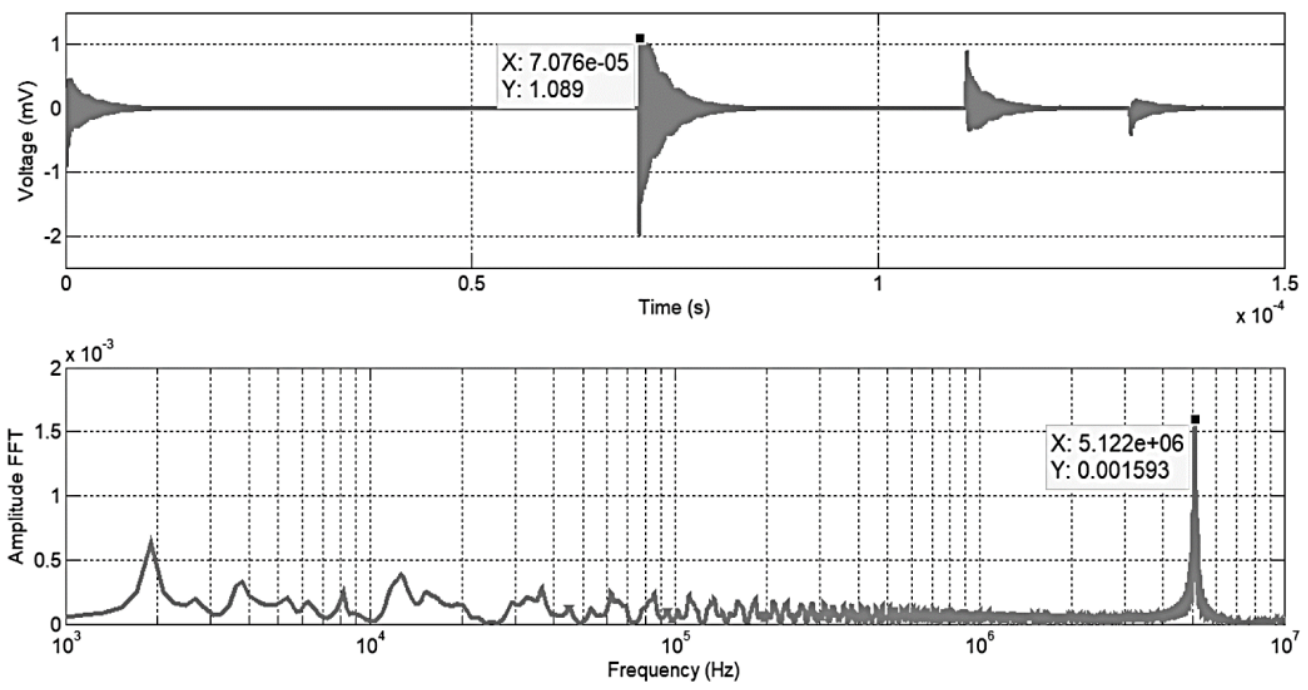

Figure 7. PD signal in time and frequency domain detected at point $\mathrm{P}(7 \mathrm{~km})$
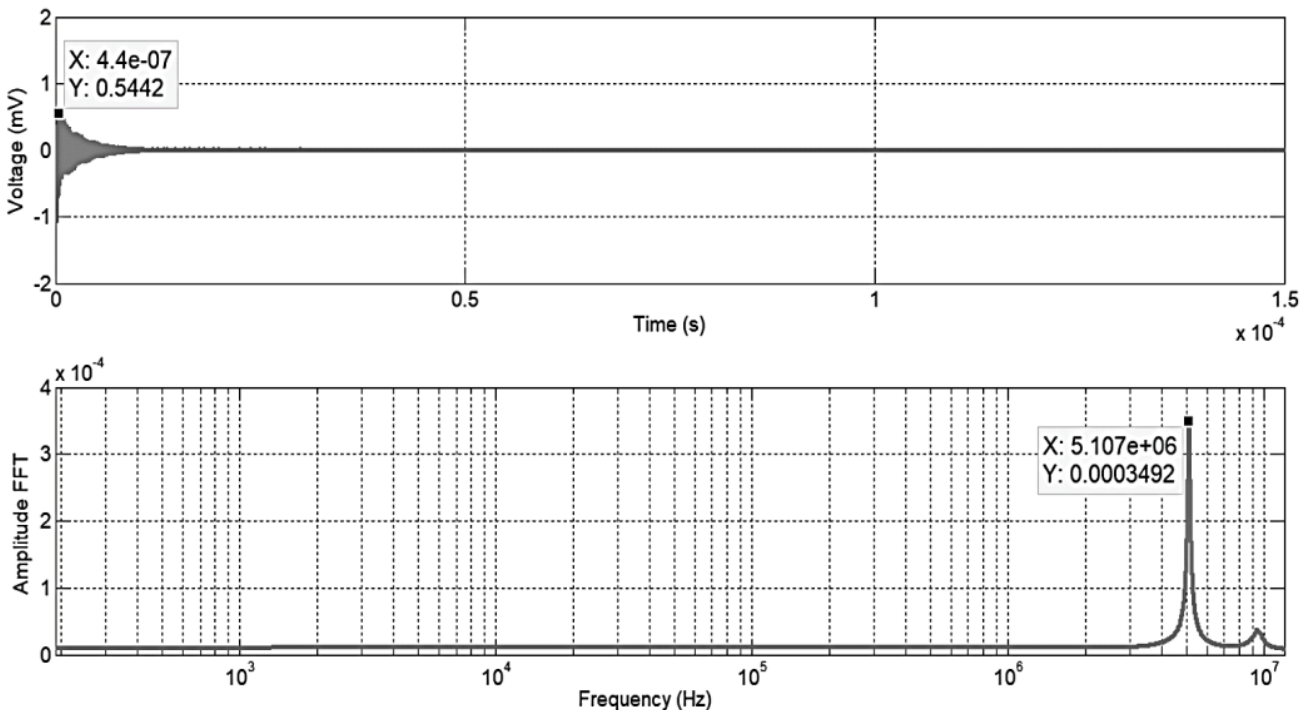

Figure 8. PD signal in time and frequency domain detected at point $S(15.5 \mathrm{~km})$

Table 2. Result of Simulations

\begin{tabular}{cccc}
\hline Location & Distance & Amplitude $(\mathrm{mV})$ & Amplitude FFT \\
\hline $\mathrm{P}$ & $11 \mathrm{~km}$ & 1.089 & 0.001593 \\
$\mathrm{Q}$ & $7 \mathrm{~km}$ & 2.634 & 0.002284 \\
$\mathrm{R}$ & $1.5 \mathrm{~km}$ & 13.13 & 0.01271 \\
$\mathrm{~S}$ & $15.5 \mathrm{~km}$ & 0.5442 & 0.0003492 \\
\hline
\end{tabular}

The step-by-step conversion of the apparent PD load measured using the MATLAB Simulink model is shown in Figure 9. The input data Figure 9 (a) in the $\mathrm{mV}$ value is imported from the measurement data to the model in Figure 3. Then filtering process using the Butterworth type is used at the next stage in Figure 9 (b) to eliminate noise. Two stages of integral are used to define the average value of the PD signal as shown in Figures 9 (c) and (d). Finally, the significant charging unit set out in Figure 9 (e) represents the charging unit in Pico Coulomb (pC) that meets the requirements of this work. The last value of the apparent charge in the measured cable is about $2500 \mathrm{pC}$, indicating the high enough of the discharge activity. From this, the correct PD signal can be identified or the noise signal has been detected. 


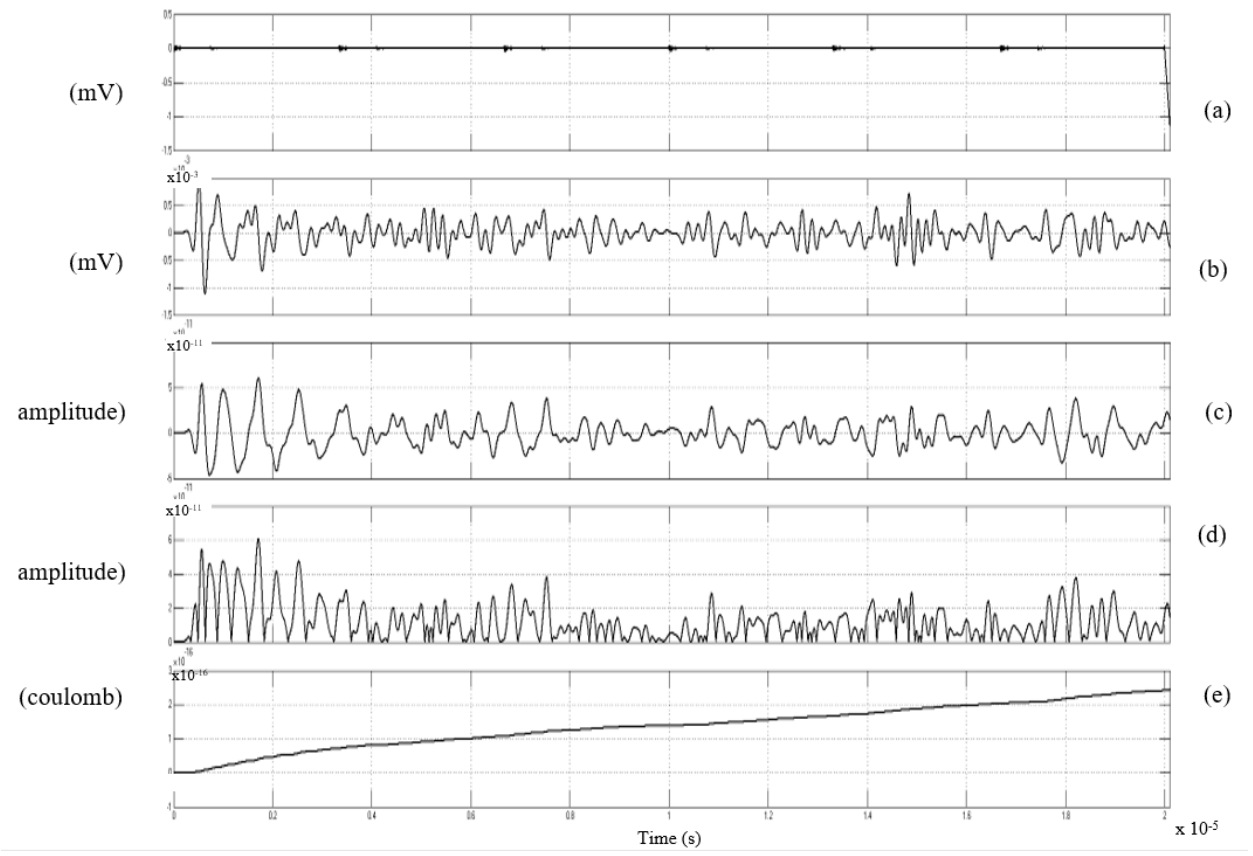

Figure 9. Apparent charges of PD measurements using MATLAB Simulink, (a) Input data in mV, (b) After filtering, (c) After first integral, (d) After second integral, (e) Apparent charge output

\section{CONCLUSION}

This paper presents the modelling and simulation of the online partial discharge (PD) measurement of the medium voltage (MV) power cable. The objective of checking the ability of RC to detect PD activity in a real distribution network has been successfully archived. The model of the RC sensors, the PD pulse and the actual $70 \mathrm{~km}$ network function properly during simulation produces a clear and significant result. It shows that the PD signal in the power cable modelled along the network can be triggered by the result of the RC sensor. The PD amplitude detected was at the highest of $13.13 \mathrm{mV}$ and the lowest at $0.54 \mathrm{mV}$, depending on the location of the RC and the PD pulse. It was clearly indicated that the nearest RC to the PD pulse would produce the highest amplitude and frequency response. Finally, the modelling of PD measurement can be further improved and explore since it gave a significant result. Finally, the modelling of the PD measurement can be further improved and explored as it has yielded significant results. This will make the actual measurement easier to perform and will help improve the ability to detect PD in the medium voltage power cable.

\section{ACKNOWLEDGEMENTS}

The authors are grateful to Universiti Malaysia Perlis (UniMAP) for the equipment support, and to the Ministry of Higher Education for their support through the SLAB scheme.

\section{REFERENCES}

[1] C. Chou and C. Chen, "Measurement and Analysis of Partial Discharge of High and Medium Voltage Power Equipment," 2018 7th Int. Symp. Next Gener. Electron., no. Isne, pp. 1-4, 2018.

[2] M. Shafiq, G. A. Hussain, L. Kütt, N. I. Elkalashy, and M. Lehtonen, "Partial discharge diagnostic system for smart distribution networks using directionally calibrated induction sensors," Electr. Power Syst. Res., vol. 119, pp. 447-461, 2015.

[3] A. Milioudis, G. Andreou, and D. Labridis, "Electrical Power and Energy Systems On-line partial discharge monitoring system for underground MV cables - Part II : Detection and location," Electr. Power Energy Syst., vol. 109, pp. 395-402, 2019.

[4] J. Fuhr, M. Haessig, and R. A. King, "Detection and Location of Internal Defects in tlie Insulation of Power Transformers," vol. 28, no. 6, pp.1057-1067, 1993.

[5] A. N. Milioudis and D. P. Labridis, "Modelling for on-line partial discharge monitoring on MV cables by using a modified Universal Line Model," 2015 IEEE Eindhoven PowerTech, PowerTech 2015, pp. 1-6, 2015.

[6] R. Schwarz and M. Muhr, "Modern technologies in optical partial discharge detection," Annu. Rep. - Conf. Electr. Insul. Dielectr. Phenomena, CEIDP, pp. 163-166, 2007. 
[7] R. N. Wu and C. K. Chang, "The use of partial discharges as an online monitoring system for underground cable joints," IEEE Trans. Power Deliv., vol. 26, no. 3, pp. 1585-1591, 2011.

[8] G. C. Montanari, D. Fabiani, P. Morshuis, and L. Dissado, "Why residual life estimation and maintenance strategies for electrical insulation systems have to rely upon condition monitoring," IEEE Trans. Dielectr. Electr. Insul., vol. 23, no. 3, pp. 1375-1385, 2016.

[9] C. Sumereder and M. Muhr, "Estimation of Residual Lifetime - Theory and Practical Problems," vol. 1, pp. 1-6, 2005.

[10] W. McDermid, "IEEE guide for the measurement of partial discharges in AC electric machinery," in Conference Record of IEEE International Symposium on Electrical Insulation, 2012, vol. 2014, p. 659.

[11] L. W. Van Veen, "Comparison of measurement methods for partial discharge measurement in power cables," no. April, pp. 1-116, 2014.

[12] H. A. Illias et al., "Measurement and simulation of partial discharge activity within a void cavity in a polymeric power cable model," pp. 105-108, 2013.

[13] D. Clark, R. Mackinlay, R. Giussani, L. Renforth, and R. Shuttleworth, "Partial Discharge Pulse Propagation, Localisation and Measurements in Medium Voltage Power Cables," 48th Int. Univ. Power Eng. Conf., pp. 1-6, 2013.

[14] P. Mulroy, A. Hurtado, and D. Badetz, "On-line partial discharge monitoring system for distribution networks," Proc. 2012 IEEE Int. Conf. Cond. Monit. Diagnosis, C. 2012, pp. 542-545, 2012.

[15] D. A. Genutis, "Neta World-Using PD surveys to increase electrical reliability," 2001.

[16] A. S. Kumar, R. P. Gupta, K. Udayakumar, and a. Venkatasami, "Online partial discharge detection and location techniques for condition monitoring of power transformers: A review," 2008 Int. Conf. Cond. Monit. Diagnosis, pp. 927-931, 2008.

[17] V. K. Fasil and S. Karmakar, "Modeling and Simulation Based Study for On-line Detection of Partial Discharge of Solid Dielectric," 2012 IEEE 10th Int. Conf. Prop. Appl. Dielectr. Mater., pp. 1-5, 2012.

[18] IEC 60270, "High-voltage test techniques - Partial discharge measurements," IEC Standar 60270, vol. 2000, 2000.

[19] H. N. Mohd Shah et al., "Develop and implementation of PC based controller for humanoid robot using digital potentiometer," Indones. J. Electr. Eng. Comput. Sci., vol. 15, no. 1, p. 104-112, 2019.

[20] N. A. Salim, H. Mohamad, Z. M. Yasin, N. Fadilah, and A. Aziz, "Graphical user interface based model for transmission line performance implementation in power system," vol. 16, no. 1, pp. 92-100, 2019.

[21] N. Telagam, S. Lakshmi, and K. Nehru, "Ber analysis of concatenated levels of encoding in GFDM system using labview," Indones. J. Electr. Eng. Comput. Sci., vol. 14, no. 1, pp. 80-91, 2019.

[22] M. N. K. H. Rohani et al., "Evaluation of Rogowski coil sensor performance using EMTP-ATP software," in 2016 3rd International Conference on Electronic Design, ICED 2016, pp. 446-451, 2016.

[23] A. Z. Bin Abdullah, M. Isa, M. N. K. H. Rohani, S. A. B. S. Jamalil, A. N. N. Abdullah, and N. Azizan, "Development of smart online partial discharge monitoring system for medium voltage power cable," Int. J. Power Electron. Drive Syst., vol. 10, no. 4, pp. 2190-2197, 2019.

[24] N. Shahar, N. F. Ghazali, N. Yahya, and M. Amir, "Common Sport Movement Recognition From Wearable Inertial Sensor," Indones. J. Electr. Eng. Comput. Sci., vol. 10, no. 1, pp. 401-413, 2018.

[25] A. Z. Abdullah and M. Isa, "Modelling of Partial Discharge Measurement Using Rogowski Coil as New Approach," in eProceedings National Innovation and Invention Competition Through Exhibition 2018, 2018.

[26] M. N. K. H. Rohani, M. Isa, M. Syahril, C. C. Yii, A. S. Rosmi, and B. Ismail, "Sigma-Delta ADC Topology Implementation Based on Partial Discharge Detection using Rogowski Coil Sensor," J. Phys. Conf. Ser., vol. 1019, no. $1,2018$.

[27] A. Z. Abdullah, M. N. K. H. Rohani, M. Isa, H. Hamid, S. N. M. Arshad, and M. Othman, "Real On-Site Partial Discharge Measurement Technique in Medium Voltage Power Cable," 2018 IEEE 7th Int. Conf. Power Energy, PECon 2018, pp. 405-408, 2018.

[28] M. Isa, "Partial Discharge Location Technique for Covered-Conductor Overhead Distribution Lines," Aalto University, p.133, 2013.

[29] IEEE Standards, "IEEE Guide for Partial Discharge Testing of Shielded Power Cable Systems in a Field Environment," IEEE Power Engineering Society, February. 2007.

[30] G. M. Hashmi, "Partial Discharge Detection for Condition Monitoring of Covered-Conductor Overhead Distribution Networks using Rogowski Coil," Doctoral Dissertation, 2008.

\section{BIOGRAPHIES OF AUTHORS}

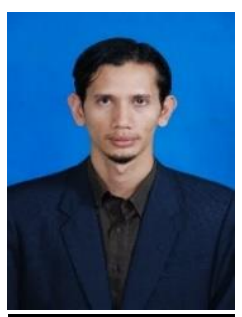

Ahmad Zaidi Bin Abdullah was born in Kedah, Malaysia, in 1981. He received his B.Eng (Hons.) degree in Industrial Electronic Engineering from the University Malaysia Perlis and his M.Eng degree in Electrical Power from the University Tenaga Nasional. He is currently pursuing a Ph.D. at Universiti Malaysia Perlis. He is currently pursuing a Ph.D. in Universiti Malaysia Perlis. He is a member of the Board of Directors of Engineer Malaysia and Institute Engineer Malaysia. His main research interest is high-voltage engineering, power transformer, and on-line partial discharge monitoring. 

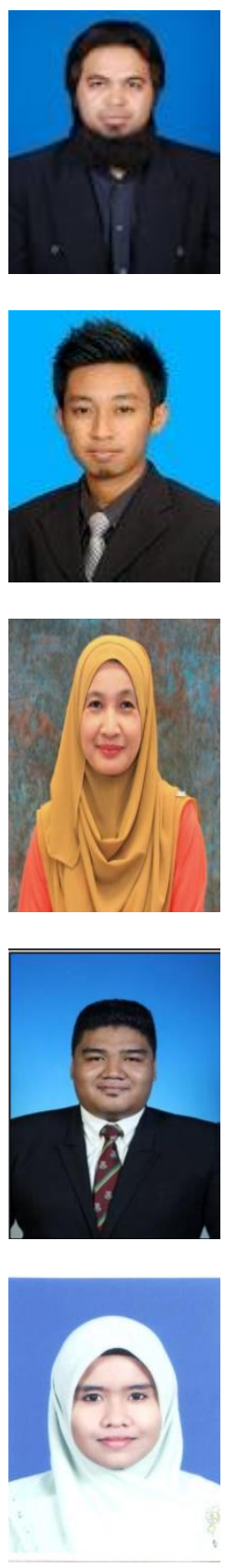

Muzamir Bin Isa was born in Perlis, Malaysia, in 1979. He received a B.Eng (Hons.) degree in Electrical Engineering from University Teknologi Malaysia, a M.Eng in Electrical Power from University Tun Hussein Onn Malaysia, and a Ph.D. in Power Systems and High Voltage Engineering from Aalto University, Finland. He is a member of the board of engineers of Malaysia. Its main research interest is high-voltage engineering, power transformer, and on-line partial discharge monitoring.

Mohamad Nur Khairul Hafizi Rohani was born in 1989 in Johor, Malaysia. He has received the B.Eng. (Hons.) Degree in Industrial Electronic Engineering and Ph.D. from University Malaysia Perlis, Malaysia, 2013. His research interests are the design of sensors based on partial discharge measurements for online condition components.

Haziah Binti Abd Hamid received her first and master's degrees in Electrical Engineering from Universiti Teknologi Malaysia (UTM) in 1999 and 2002 respectively. She received a Ph.D. in Electrical and Electronic Engineering from Cardiff University, UK, in 2012. She is a senior lecturer and currently holds the position of Dean at the School of Electrical Systems Engineering, Universiti Malaysia Perlis, Malaysia. Her main research interests include high voltage transients, insulation systems and earthing systems.

Muhammad Harith Bin Amlus is a lecturer at the School of Business and Technology at the University Malaysia Perlis. He received a Ph.D. in Management and a Masters in Science Technology from Universiti Utara Malaysia (UUM), B.Eng. (Hons.) Manufacturing at Universiti Teknikal Malaysia. His research interests lie in the re-engineering and innovation of manufacturing industries process.

Nurhafiza bt Azizan was born in 1982 in Pulau Pinang, Malaysia. She's received a B.Eng. (Hons.) Degree in Industrial Electronic Engineering and Master's degree in Electrical Power Engineering from University Malaysia Perlis, Malaysia. She has worked at the Faculty of Engineering Technology as a Vocational Training Official. Registered as Professional Technology (Ts), her research interests include power systems and energy management systems. 